\title{
3D-Printed transmit-array antenna for broadband backhaul 5G links at V-band
}

\author{
Sérgio A. Matos, Senior Member, IEEE, Jorge P. Teixeira, Student Member, Jorge R. Costa, Senior \\ Member, IEEE, Carlos A. Fernandes, Senior Member, IEEE, Nour Nachabe, Cyril Luxey, Fellow, \\ IEEE, Diane Titz, Senior Member, IEEE, Frederic Gianesello, Member, IEEE, Carlos del Rio, Senior \\ Member, IEEE, Ana Arboleya, Member, IEEE, Jean-Philippe Garnero, Jean-François Vizzari
}

\begin{abstract}
The low-cost and compactness of transmit-array antennas (TAs) make them attractive for $5 \mathrm{G}$ backhaul links. However, the TA advantage is less obvious when considering the broadband operation requirement. Two main factors influence the bandwidth performance: i) the bandwidth of the unit cells; ii) the number of $360^{\circ}$ phase wrapping zones in the aperture, which are designed for a specific frequency. Herein, we overcome these limitations by using all-dielectric unit cells (inherently broadband) and by developing a general method to quantify and manage the intricate relation between antenna gain, bandwidth and antenna height. Based on this framework we optimize, as an example, a TA design (focal distance, $F=63 \mathrm{~mm}$ and aperture diameter $D=$ $80 \mathrm{~mm}$ ) to comply with typical gain specification for $5 \mathrm{G}$ backhaul links ( $>30 \mathrm{dBi}$ ) in the WiGiG band (from 57 to $66 \mathrm{GHz}$ ). The feed is a dedicated compact horn $\left(8 \times 5 \times 22 \mathrm{~mm}^{3}\right)$ that provides a proper illumination of the aperture. Additive manufacturing is used to simplify the manufacturing process of the antenna. A very good agreement between simulations and experimental results is obtained, achieving good aperture efficiency for this type of antenna $(42 \%)$, which rivals with existing solutions based on more expensive manufacturing techniques.
\end{abstract}

Index Terms-5G backhaul link, WiGiG band, TransmitArray, V-band Horn antenna, Additive manufacturing

\section{INTRODUCTION}

$\mathrm{T}$ HE massification of $5 \mathrm{G}$ will pivot on cost-effective technologies that can support the development of the Enhanced Mobile Broadband (eMBB) platform. In the context of pico and femto cells, that will constitute the landscape of future 5G mobile communications, extremely low-cost backhaul links between base stations and access points are required. Millimeter-waves $(\mathrm{mmW})$ provide the necessary throughputs with extremely low deployment cost when compared with fiber optics-based backhaul solutions. In particular, the WiGiG band from 57 to $66 \mathrm{GHz}$, offers large

Manuscript received October, 2019. This work was partially supported by grant ISTA-BM-2016 and partially supported by Fundação para a Ciência e Tecnologia (FCT) under projects PTDC/EEI-TEL/30323/2017 (“ADAM3D") and UIDB/EEA/50008/2020.

Sérgio A. Matos, Jorge R. Costa, Jorge P. Teixeira and Carlos A. Fernandes are with Instituto de Telecomunicações, Instituto Superior Técnico, Universidade de Lisboa, Av. Rovisco Pais 1, 1049-001 Lisboa, Portugal (Email: segio.matos@1x.it.pt; Jorge.costa@1x.it.pt; Carlos.Fernandes@1x.it.pt)

Jorge R. Costa, Jorge P. Teixeira and Sérgio A. Matos are also with Instituto Universitário de Lisboa (ISCTE-IUL), Departamento de Ciências e bandwidths over unlicensed spectrum and high frequency reuse capability, ideal in dense cell environments [1]. The electronics industry is responding to this current need of increasing the operating frequency by providing new chipset $\mathrm{mmW}$ antennamodules [2]-[4]. Phased-array configurations could be an alternative, but they lead to excessive feeding network losses and their cost scales with gain and frequency [5], [6] as well as DC consumption. Progress has been made on commercial integrated high gain antenna solutions for V-band [7], however, the cost of these antennas is still the main bottleneck. High fabrication precision is required to meet the tolerances, which impacts the antenna cost. Therefore, the antenna design should also aim for a simple fabrication process. The ongoing development of additive manufacturing can offer new possibilities for cost reduction. For example, dielectric lensbased solutions can benefit from the low-cost of fused deposition modeling (FDM) technique, or injection molding in mass production. The drawback is that materials used in these technologies tend to be lossy, such as ABS-M30 ( $\varepsilon_{r}=$ $2.48, \tan \delta=0.009)$ ).

An intensive research effort is being carried out to achieve a solution that can deliver high gain (typically above $30 \mathrm{dBi}$ [1]) in the entire WiGiG band [8], [9] with the lowest possible cost. In [8], the authors integrated a 3D printed elliptical chopped dielectric lens with a FR4 PCB antenna-source to favor gain enhancement at low-cost. However, the gain performance of this solution was limited by the radiation efficiency of the FR4 primary feed antenna and by the dielectric losses of the lens material. In [10], the authors showed that those losses could be reduced by using a transmit-array (TA) lens - sometimes designated as discrete dielectric lens (DDL). We showed that the antenna radiation efficiency increased from $60 \%$ to $83 \%$ when a PLA chopped elliptical lens was replaced by an equivalent PLA TA. Other 3D printed DDL designs can be found in the literature [11]-[17]. Achieving high gain is quite

Tecnologias da Informação Av. das Forças Armadas, 1649-026 Lisboa, Portugal (Jorge Pedro Teixeira@iscte-iul.pt)

Nour Nachabe, C. Luxey, Diane Titz, Jean-Philippe Garnero and JeanFrançois Vizzari are with Polytech'Lab, Université Nice Sophia Antipolis, 06560 Valbonne, France.

F. Gianesello, is with STMicroelectronics, 38920 Crolles, France.

Carlos del Rio is with Universidad Pública de Navarra, UPNA, Pamplona, Spain

Ana Arboleya is with with Universidad Rey Juan Carlos, Madrid, Spain. 
straightforward, however, the challenge is to overcome the bandwidth limitation caused by the number of $360^{\circ}$ phase jump zones that directly correlates with the $F / D$ ratio. In [13] the bandwidth problem was solved by increasing the focal distance until lens zoning disappears. This leads to large focal lengths $(F / D=2.8)$ which impacts on the antenna height. A clever scheme is proposed in [13] to reduce the focal distance by a factor of 3 using a polarization rotation reflection surface and polarization grid. However, these antennas become inherently single polarization and the additional complexity of the configuration impacts on the antenna-system and terminal cost.

In this work, we present a design procedure for dielectric TAs antennas (or DDL) that optimize the compromise between $F / D$ ratio, gain and bandwidth. We use our guidelines to show that an all-dielectric TA antenna can be a viable affordable option to meet the specifications for broadband backhaul connections at V-band. The unit cell design procedure of TAs provides a fresh perspective into the classical topic of Fresnel Zone Plate Lens [14]. Applying the same approach used in [23] to evaluate the scanning performance of a metal-based multilayered TA, we demonstrate that it is possible to achieve a closed-form expression that accurately captures the relation between gain, bandwidth and focal distance. The simplicity of this method contrasts with the complexity of the $\mathrm{GO} / \mathrm{PO}$ integral formulation used in [15] for FZPL. Furthermore, it provides a direct relation between gain and bandwidth that is not captured by the usual approach of imposing a maximum phase error criteria [16].

A compact 3D-printed metal-coated polymer corrugated horn antenna is also specially designed to provide proper illumination of the lens. Metal 3D printed corrugated horns exhibit good performances at $\mathrm{W}$ band or even up to $300 \mathrm{GHz}$ [18]-[20], but the metal coating of 3D-printed polymer parts is a more affordable technique [20]. As the frequency increases, this process becomes more changeling due to the increase of sensitivity to the surface roughness and coating conductivity, but thanks to the current evolution of this technology [21], it is now a cost-effective alternative for V-band.

This demonstrates that the whole antenna production can be based on additive manufacturing, leading to a compact $(F / D=$ $0.8)$ antenna system at the $\mathrm{V}$-band with high gain $(>30 \mathrm{dBi})$ and good aperture efficiency $(42 \%)$.

In section II, the design guidelines for a DDL are provided based on a GO/PO analysis. In section III, we implement these guidelines to design an antenna (feed and lens) compliant with the gain specifications for WiGiG 5G backhaul links. Finally, section IV addresses the measured results.

\section{DIELECTRIC TRANSMIT-ARRAY}

In a TA each portion of the aperture acts as a spatial phase shifter - the unit cell - that adds to the incoming phase $\phi_{i n}$, a prescribed phase delay $\phi_{\text {lens }}$, such that $\phi_{\text {out }}=\phi_{\text {in }}+\phi_{\text {lens }}$. The unit cells are designed as if the rays passing through the lens are perpendicular to its surface, which can be a reasonable approximation for low lens thicknesses and low incidence angles (Fig. 1). In this work, the unit cell is a parallelepiped with height $h_{\max }$, composed of a dielectric (ABS-M30) with height $h$, and air (occupying the remaining volume). The control of the unit cell phase shift is obtained by varying the dielectric height $h$, where $\varepsilon_{r}$ is the real part of the dielectric permittivity and $k_{0}$ is the free-space wavenumber. Considering a minimum height different from zero avoids having a lens composed by separated annular regions. Herein, we consider $h_{\min }=1 \mathrm{~mm}$, which is enough for the lens structural integrity. On the other hand, the height $h_{\text {max }}$ is fixed to provide the usual $360^{\circ}$ phase span in TA design for a given wavelength $\lambda_{0}$. The aperture is designed to produce boresight collimation by distributing the unit cells according to the profile

$$
h(r)=h_{0}+\frac{F-\Delta l+m \lambda_{0}}{\sqrt{\varepsilon_{r}}-1}, R_{m}<r<R_{m+1}
$$

Where $h_{0}$ is the central height of the lens $(r=0), \Delta l=$ $\sqrt{r_{i}^{2}+F^{2}}-F$ (see Fig. 1) and $m$ defines the zone of the lens up to a total of $K$ zones. The radial positions of the transitions between zones are defined by the condition $h\left(R_{m}\right)=h_{\text {min }}$. We considered the typical half wavelength unit cell size, $w=$ $2.5 \mathrm{~mm}\left(\frac{\lambda_{0}}{2} @ 60 \mathrm{GHz}\right)$ resulting in the stepwise lens profile given in Fig. 1. The radial symmetry of this problem allows assembling the lens using concentric annuli with fixed widths $w$, as represented in the inset of Fig. 1. The diffraction effects are then reduced when compared with a fully pixelized version of the lens.

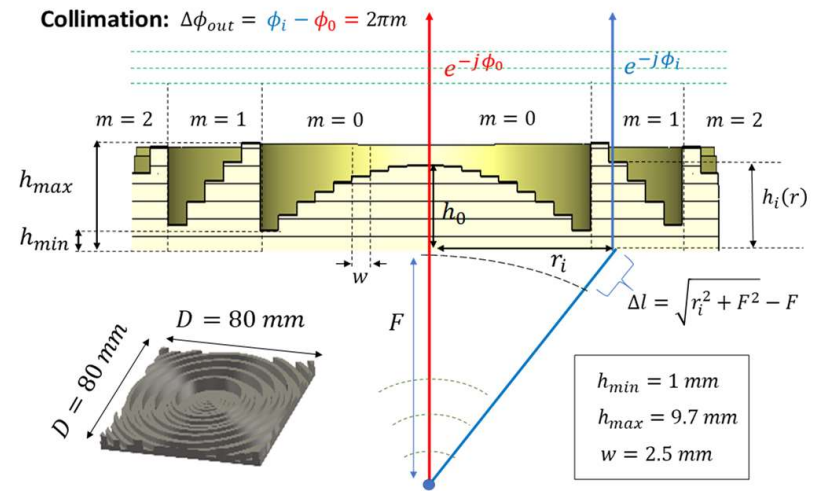

Fig. 1. Working principle of a dielectric transmit-array for beam collimation.

The outgoing electric field from the TA in a plane of constant phase (green dashed lines in Fig. 1) can be approximated by Gaussian amplitude distribution with standard deviation $\sigma$

$$
\vec{E}_{a b}(r) \approx e^{-\frac{r^{2}}{2 \sigma^{2}}} e^{j \Phi_{o u t}} \vec{u}_{E} .
$$

where $\Phi_{\text {out }}$ varies with wavelength as a stepwise function

$$
\Phi_{\text {out }}=-2 \pi m \frac{\lambda_{0}}{\lambda}, R_{m}<r<R_{m+1} .
$$

With $m=\{0,1, \ldots, K\}$. The standard deviation $\sigma$ is related to the taper level, $\tau_{d B}, E_{a b}(r=D / 2)=10^{-\tau_{d B} / 20}$. The typical taper value is $\tau_{d B}=10 \mathrm{~dB}$ which ensures a good compromise between losses due to the spillover and sub-illumination of the aperture. As the position of the $360^{\circ}$ phase transitions depends on the operating frequency, phase errors build-up as the number of lens zones increases. Considering the Gaussian 
approximation, the integration of the fields of the aperture can be done analytically using GO/PO analysis. The directivity for the boresight direction can then be calculated explicitly

$$
D_{\max }=D_{\text {ir } 0}\left|\frac{\sum_{m=0}^{K} e^{-j \frac{2 \pi m \lambda_{0}}{\lambda}}\left(e^{\left.-\frac{R_{m}^{2}}{2 \sigma^{2}}-e^{-\frac{R_{m+1}^{2}}{2 \sigma^{2}}}\right)}\right.}{1-e^{-\frac{D^{2}}{4 \sigma^{2}}}}\right|^{2} .
$$

Where

$$
D_{i r 0}=s_{T A}\left(\frac{4 \pi \sigma}{\lambda}\right)^{2} \tanh \left[\left(\frac{D}{4 \sigma}\right)^{2}\right]
$$

is the directivity of a circular aperture with constant phase $(\lambda$ $=\lambda_{0}$ ) and Gaussian illumination, as in Eq.8 of [23]. $R_{m}$ is defined by $h_{i}\left(R_{m}\right)=h_{\min }$ with $m=\{0,1, \ldots, K\}$ and since the last zone can be truncated, $R_{K+1}=D / 2$. We introduce the parameter $s_{T A}$ as the TA scaling factor that accounts for other effects that were not considered in this $\mathrm{GO} / \mathrm{PO}$ analysis, such as unit cell reflection and insertion losses, aperture illumination spillover and phase errors caused by refraction and diffraction (which are more significant near the lens $360^{\circ}$ phase transitions). Looking at a wide variety of different types of TAs, $s_{T A}$ is typically below $50 \%$ [23]. Therefore, we considered $s_{T A}=3 \mathrm{~dB}$ for the design process. As shown in [10], the radiation efficiency of these dielectric TAs is high, allowing to approximate the antenna gain by its directivity. The aperture dimension and the focal distance can then be defined from a given gain and bandwidth criteria according to (4) and (5).

\section{DESIGN OF A TRANSMIT-ARRAY LENS FOR WiGiG BAND}

An antenna for $5 \mathrm{G}$ backhaul wireless links needs to deliver high gain, typically above $30 \mathrm{dBi}$ [1]. WiGiG band usually refers to the [57-66] GHz band according to 802.11.ad standard, but recently an extended WiGiG band was defined (802.11.ay) covering [57-70] GHz (see inset of Fig. 2)

\section{A. TA design}

We set a $31 \mathrm{dBi}$ directivity $D_{0}$, to give a $1 \mathrm{~dB}$ margin to accommodate the bandwidth limitation of the TA in the specified gain criteria. The aperture dimension follows from : $D_{0}(D)=31(\mathrm{dBi}) \rightarrow D \approx 80(\mathrm{~mm})$. The maximum number of phase transitions is determined by the $F / D$ ratio, where $F$ is the focal distance. For this aperture size, we use equation to estimate the minimum focal distance that complies with the 30 $\mathrm{dBi}$ minimum gain criteria of the $\mathrm{WiGiG}$ band. By setting $h_{0}=$ $h_{\max }$ the radius of the first lens zone $(m=0)$, which has no bandwidth limitation, is maximum. Neglecting second order effects, this condition maximizes the lens bandwidth as well. Later, full-wave simulations are employed to tune this parameter, further optimizing the gain and lens bandwidth. In this case, the phase transition occurs at $R_{m}=$ $\sqrt{m^{2} \lambda_{0}^{2}+2 F \lambda_{0} m}$. In Fig. 2 we plot for several $F / D$ ratios and select the minimum value that complies with the gain criteria for the extended WiGiG band, corresponding to $F / D=0.8$. Fig. 2 captures the interplay between the dependence of $D_{\max }$ with frequency and the gain degradation due to the phase error caused by the zoning process. For $F / D>2$ no zoning occurs $(K=0)$ thus $D_{\max }=D_{0}$.

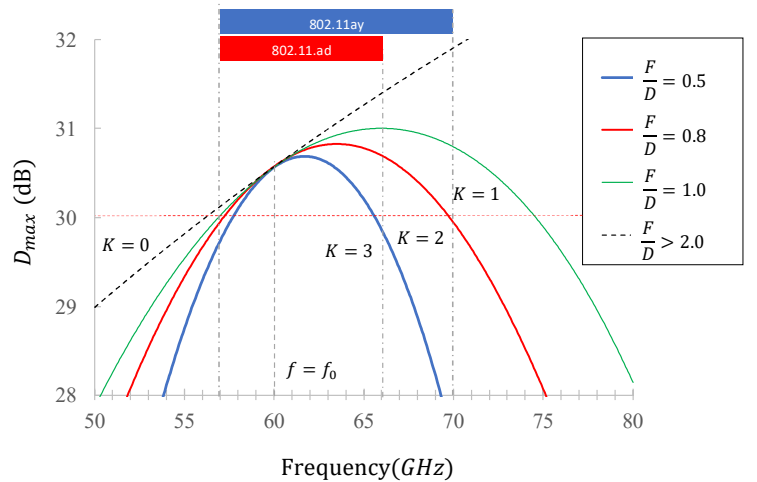

Fig. 2. $\mathrm{PO} / \mathrm{GO}$ directivity $D_{\max }$ variation with frequency, Eq. , for different focal distances considering $D=80 \mathrm{~mm} \tau_{\mathrm{dB}}=10 \mathrm{~dB}$ and $s_{T A}=3 \mathrm{~dB}$

\section{B. Feed performance}

According to the designed DDL lens, a focal ratio $F / D=0.8$ and $\tau_{d B}=10 \mathrm{~dB}$ taper level implies a $13 \mathrm{dBi}$ feed directivity [23]. A dedicated compact corrugated horn antenna was designed and fabricated $(8 \times 5 \times 22 \mathrm{~mm})$. It is wideband and has a stable radiation pattern in the WiGiG band $(57-66 \mathrm{GHz})$. The performance of this design is shown in Fig. 3. A $13 \mathrm{dBi}$ copol gain was achieved in the entire WiGiG band from 56 to 67 $\mathrm{GHz}$, with high radiation efficiency $(>85 \%)$, low cross polarization $(>27 \mathrm{~dB})$, and good polarization purity $(>27 \mathrm{dBi})$. Measured and simulated gains agree very well.

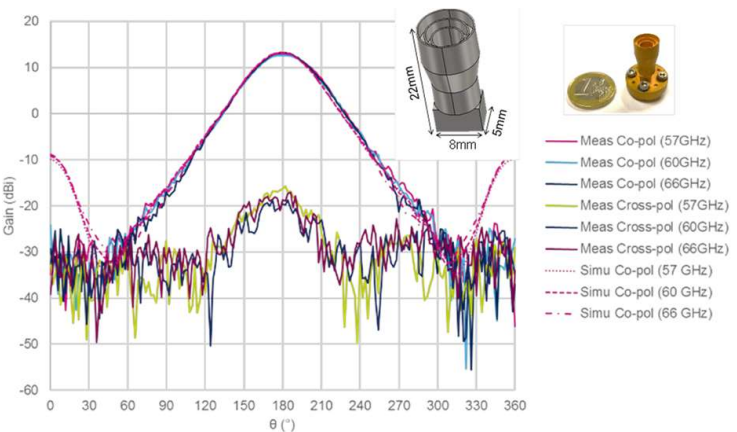

Fig. 3. Radiation patterns of the manufactured horn antenna .

\section{AnTENNA MEASUREMENTS}

The measurement campaign for the radiation patterns was conducted in our in-house measurement system: a spherical measurement range capable of performing amplitude-only acquisitions up to $140 \mathrm{GHz}$ at fixed distances of $20 \mathrm{~cm}, 40 \mathrm{~cm}$ and $60 \mathrm{~cm}$ (Fig. 4 a). Considering the main diagonal of the lens, the far-field (FF) distance at $66 \mathrm{GHz}$ is $6.13 \mathrm{~m}$. Thus, it is necessary to resort to near-field to far-field (NF-FF) transformation techniques to obtain the radiation pattern. The employed phaseless NF-FF algorithm is based on the phaseless Sources Reconstruction Method (pSRM) [24]. The pSRM is an iterative phaseless technique which relies on the spatial variability of the NF with distance and, therefore, it requires amplitude information from two or more NF acquisition surfaces. The main advantage of iterative techniques is that they do not require any modification of the measurement setup with 
respect to a regular FF acquisition. However, there is a risk of stagnation of the algorithm due to the use of iterative solvers for nonlinear systems of equations. This risk becomes higher when the variability of the field within the NF acquisition surfaces is low, as might be the case with lens antennas, yielding less accurate FF results. The field amplitude is acquired at two spherical surfaces with radii of $40 \mathrm{~cm}$ and $60 \mathrm{~cm}$. The sampling rate is $\lambda / 4$ at the highest frequency of the band, $66 \mathrm{GHz}$, as required to perform phaseless NF-FF transformations [25]. Due to mechanical restrictions of the setup, the acquisition surfaces are truncated to a $\phi$ and $\theta$ angular range of $\left[-25^{\circ}, 25^{\circ}\right]$, yielding a valid margin of the FF data of $\left[-14^{\circ}, 14^{\circ}\right]$ in both axes [25]. In Fig. 5 the FF amplitude and phase of the copolar component of the field, computed from the NF phaseless acquisitions is compared with full-wave simulation for the E-plane of the copolar component. The agreement between measurements and simulations is good within the valid margin area, shaded in grey. The main sources of the retrieval error are related to the truncation of the acquisition planes and the accuracy of the phase retrieval method and, to a lesser extent, mechanical deviations of the robotic arms and random errors of the RF instrumentation. Finally, the antenna gain was computed by means of the well-known intercomparison method [26] with a standard gain horn. The method is adapted for NF acquisitions in order to compensate the variation introduced by the NF-FF transformation to the radiation pattern and thus, the gain is given by $G_{\text {lens }, F F}=G_{l e n s, N F}+\left(D_{\text {lens, } F F}-D_{\text {lens }, N F}\right)$, where $G_{l e n s, N F}$ is the measured gain in NF, $27.16 \mathrm{dBi}$ at $60 \mathrm{GHz}$. $D_{\text {lens,FF }}$ and $D_{\text {lens,NF }}$ are directivities from $\mathrm{FF}$ and $\mathrm{NF}$ respectively, computed from pattern integration of the extracted FF pattern by means of the pSRM and the truncated NF amplitude-only acquisitions respectively. The obtained values at $60 \mathrm{GHz}$ are 34.67 and $29.95 \mathrm{dBi}$, yielding a $\mathrm{FF}$ gain value of $31.9 \mathrm{dBi}$. The final FF gain values for the rest of the studied frequencies are gathered in Fig. 6 and compared with the results obtained from full-wave simulations and the PO/GO analysis. Based on previous measuring experiences, we estimate that the uncertainty of measured gain is $\pm 1.2 \mathrm{~dB}$ (corresponding to the error bars in Fig. 6), which is in line with the expected accuracy of these types of algorithms [24][25]. According to full-wave simulations this antenna provides a radiation efficiency of $91 \%$ with a beam efficiency of $52 \%$ at $60 \mathrm{GHz}$. As intended the gain is higher than the required $30 \mathrm{dBi}$ within the complete operating band. It is also worth mentioning that the TA does not affect the return loss of the horn. The $S_{11}$ of the complete antenna (Horn+Lens) is always below $-15 \mathrm{~dB}$ in the entire band of operation. In TABLE 1 the developed TA is compared with other recent designs. Different types of lenses are considered: full dielectric TA manufacture with FDM 3D-printing (3D FDM TA); a TA of stacked metallic layers embedded in a dielectric host medium (Metallic TA); and a Fresnel Zone Plate Lens (FZPL). We conclude that the proposed solution provides a good balance between $F / D$, gain $\left(G_{F F}\right)$ and $1 \mathrm{~dB}$ bandwidth $(1 \mathrm{~dB} B W)$ with simple design and manufacturing processes.

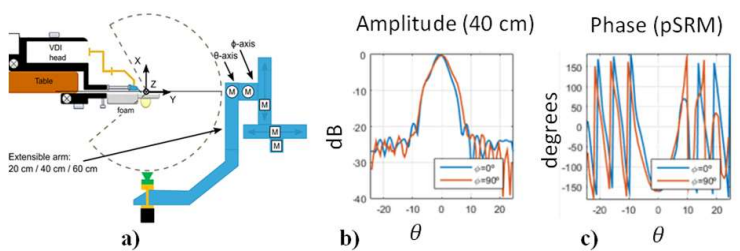

Fig. 4. (a) NF acquisition setup; NF acquired data (b) and corresponding phase using pSRM (b), along main cuts for $60 \mathrm{GHz}$ at $40 \mathrm{~cm}$.



Fig. 5. Normalized radiation pattern (E-plane) at $60 \mathrm{GHz}$.

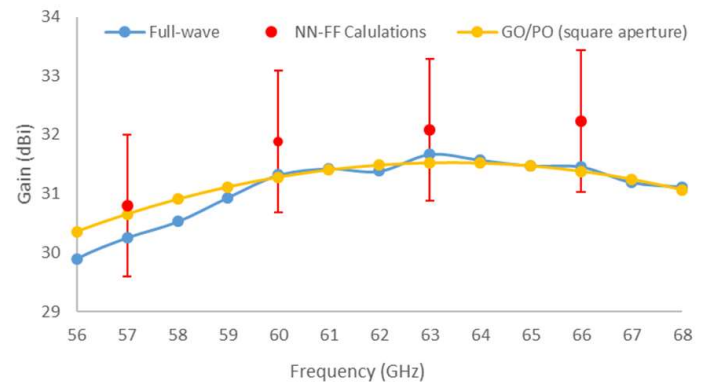

Fig. 6. - Gain comparison with $\mathrm{PO} / \mathrm{GO}$ analysis for a square aperture $\left(s_{T A}=\right.$ $2.3 d B$ in (5)), full-wave simulations and NN-FF calculation.

TABLE 1.

COMPARISON WITH PREVIOUS RELATED WORKS

\begin{tabular}{ccccccc}
\hline \hline Antenna & $\boldsymbol{f}_{\mathbf{0}}(\boldsymbol{G H z})$ & $\boldsymbol{D}\left(\boldsymbol{\lambda}_{\mathbf{0}}\right)$ & $\boldsymbol{F} / \boldsymbol{D}$ & $\begin{array}{c}\boldsymbol{G}_{\boldsymbol{F F}} \\
(\boldsymbol{d B i})\end{array}$ & $\begin{array}{c}\boldsymbol{\eta}_{\boldsymbol{a b}} \\
(\%)\end{array}$ & $\begin{array}{c}\mathbf{1 d B} \mathbf{~ B W} \\
(\%)\end{array}$ \\
$\begin{array}{c}\text { This work } \\
\text { 3D FDM }\end{array}$ & 60.0 & 16.0 & 0.78 & 31.9 & 48 & 15 \\
$\begin{array}{c}\text { TA [27] } \\
\text { 3D FDM }\end{array}$ & 60.0 & 9.50 & 0.42 & 23.0 & 18 & 16 \\
$\begin{array}{c}\text { TA [13] } \\
\text { Metallic }\end{array}$ & 60.0 & 40.0 & 2.50 & 37.5 & 36 & 43 \\
$\begin{array}{c}\text { TA [9] } \\
\text { FZPL [28] }\end{array}$ & 61.5 & 20.5 & 0.70 & 32.5 & 43 & 15 \\
\hline \hline
\end{tabular}

\section{CONCLUSIONS}

The paper introduces GENERAL design rules for controlling the operation bandwidth of TA antennas, to overcome the limitation of aperture phase-wrapping associated limitations. In known designs, the $F / D$ ratio is defined using heuristic assumptions. Herein, a closed form expression is presented to find the optimal $F / D$ ratio of the antenna according to gain and bandwidth criteria. A horn antenna for the V-band was also designed for this specific application. The antenna design privileges a simple, low-cost fabrication process based on the most affordable $3 \mathrm{D}$ printing techniques, making it a costeffective alternative to existing solutions for mm-wave highgain antennas. The presented antenna example is intended for for $5 \mathrm{G}$ backhaul communication links at $\mathrm{WiGiG}$ band. 


\section{REFERENCES}

[1] ECC Report 288: "Conditions for the coexistence between Fixed Service and other envisaged outdoor uses/applications in the 57-66 GHz range", Jan. 2019. https://www.ecodocdb.dk/document/category/ECC Reports

[2] https://perasotech.com/press-releases/peraso-launches-802-11admodules-wireless-backhaul/

[3] A. Bisognin et al., "Ball Grid Array Module With Integrated Shaped Lens for $5 \mathrm{G}$ Backhaul/Fronthaul Communications in F-Band," in IEEE Transactions on Antennas and Propagation, vol. 65, no. 12, pp. 63806394, Dec. 2017.

[4] C. Belem-Goncalves et al., "300 GHz quadrature phase shift keying and QAM16 56 Gbps wireless data links using silicon photonics photodiodes," in Electronics Letters, vol. 55, no. 14, pp. 808-810, 1172019.

[5] U. Rüddenklau, "mmWave Semiconductor Industry Technologies: Status and Evolution," ETSI white paper $\mathrm{N}^{\circ} 15$ second edition (2018).

[6] https://www.rfmw.com/Products/Detail/QPF4001Qorvo/631506/?utm_source=MJ\%20Product $\% 20 \mathrm{e} N$ Newsletter $\% 204-24$ $19 \& u t m$ medium $=$ email\&utm campaign $=$ Qorvo $\% 20$ coop\&utm term=Qorvo\&utm content=product $\% 20$ page

[7] L. Dussopt et al., "A V-Band Switched-Beam Linearly Polarized Transmit-Array Antenna for Wireless Backhaul Applications," in IEEE Transactions on Antennas and Propagation, vol. 65, no. 12, pp. 67886793, Dec. 2017.

[8] N. Nachabe, C. Luxey, D. Titz, J. R. Costa, S. A. Matos, F. Gianesello, C. A. Fernandes, "Low-cost $60 \mathrm{GHz}$ 3D printed lens fed by a planar source with WR15 transition integrated on FR4 PCB," 2017 IEEE International Symposium on Antennas and Propagation \& USNC/URSI National Radio Science Meeting, San Diego, CA, 2017, pp. 2671-2672.

[9] C. Jouanlanne, A. Clemente, M. Huchard, J. Keignart, C. Barbier, T. Le Nadan, L. Petit, "Wideband Linearly Polarized Transmitarray Antenna for $60 \mathrm{GHz}$ Backhauling," in IEEE Transactions on Antennas and Propagation, vol. 65, no. 3, pp. 1440-1445, March 2017.

[10] J. P. Teixeira, S. A. Matos, J. R. Costa, C. A. Fernandes, N. Nachabe, C. Luxey, D. Titz, , F. Gianesello, "Transmit array as a viable 3D printing option for backhaul applications at V-band," 2017 IEEE International Symposium on Antennas and Propagation \& USNC/URSI National Radio Science Meeting, San Diego, CA, 2017, pp. 2641-2642.

[11] E. Jo and D. Kim, "3-D Printer Based Lens Design Method for Integrated Lens Antennas," in IEEE Antennas and Wireless Propagation Letters, vol. 17, no. 11, pp. 2090-2093, Nov. 2018

[12] A. Massaccesi et al., "3D-Printable Dielectric Transmitarray With Enhanced Bandwidth at Millimeter-Waves," in IEEE Access, vol. 6, pp. 46407-46418, 2018.

[13] C. Fan, W. Yang, W. Che, S. He and Q. Xue, "A Wideband and LowProfile Discrete Dielectric Lens Using 3-D Printing Technology," in IEEE Transactions on Antennas and Propagation, vol. 66, no. 10, pp. 51605169, Oct. 2018.

[14] H. D. Hristov, Fresnel Zones in Wireless Links Zone Plate Lenses and Antennas, London, U.K.:Artech House, 2000.

[15] D.N. Black, J. C. Wiltse, "Millimeter-Wave Characteristics of PhaseCorrecting Fresnel Zone Plates," in Transactions on Microwave Theory and Techniques, vol. 35 , no. 1, Dec. 1987

[16] S. Silver, Microwave Antenna Theory and Design. Peter Pereginus, London, 1984.

[17] K. H. Jeong and N. Ghalichechian, "Design, Fabrication and Measurement of a Millimeter Wave Fresnel Lens using Additive Manufacturing," 2018 IEEE International Symposium on Antennas and Propagation \& USNC/URSI National Radio Science Meeting, Boston, MA, 2018, pp. 1879-1880.

[18] Bing Zhang, Wei Chen, Yanjie Wu, Kang Ding, and Rongqiang Li, "Review of 3D Printed Millimeter-Wave and Terahertz Passive Devices," International Journal of Antennas and Propagation, vol. 2017, Article ID 1297931, 10 pages, 2017.

[19] B. Zhang Y. Guo, H Sun, Y. Wu, "Metallic, 3D-Printed, K-Band-Stepped, Double-Ridged Square Horn Antennas," Appl. Sci., Vol.8, Issue33, 2018.

[20] J. S. Chieh, B. Dick, S. Loui and J. D. Rockway, "Development of a KuBand Corrugated Conical Horn Using 3-D Print Technology," in IEEE Antennas and Wireless Propagation Letters, vol. 13, pp. 201-204, 2014.

[21] http://www.swissto12.com/products/process/

[22] John L. Volakis, Antenna engineering handbook, New York: McGrawHill, [2007], chapter 18.

[23] E. B. Lima, S. A. Matos, J. R. Costa, C. A. Fernandes and N. J. G. Fonseca, "Circular Polarization Wide-Angle Beam Steering at Ka-Band by In-Plane
Translation of a Plate Lens Antenna," in IEEE Transactions on Antennas and Propagation, vol. 63, no. 12, pp. 5443-5455, Dec. 2015.

[24] Y. Álvarez, F. Las-Heras, M. R. Pino, "The sources reconstruction method for amplitude-only field measurements," IEEE Transactions on Antennas and Propagation, vol. 58, no. 8, pp. 2776-2781, August 2010.

[25] J. E. Hansen (1988) "Spherical Near-Field Antenna Measurements". Peter Peregrinus, London.

[26] IEEE Standard test procedures for antennas, ANSI/IEEE Std 149-1979.

[27] H. Yi, S. Qu, K. Ng, C. H. Chan and X. Bai, "3-D Printed MillimeterWave and Terahertz Lenses with Fixed and Frequency Scanned Beam," in IEEE Transactions on Antennas and Propagation, vol. 64, no. 2, pp. 442-449, Feb. 2016

[28] J. Pourahmadazar, T. A. Denidni, “Towards Millimeter-wavelength: Transmission-Mode Fresnel-Zone Plate Lens Antennas using Plastic Material Porosity Control in Homogeneous Medium," in Sci Rep, vol. 8 , no. 5300, pp. 1-14, Mar. 2018. 